\title{
Instrumentos Normativos no Atendimento Pré-Hospitalar: Enfermagem e Legislação
}

\section{Normative Instruments in Pre-Hospitalar Attendance: Nursing and Legisla- tion}

Artigo

Original

\author{
Márcia R. Braz ${ }^{1}$ \\ Reginaldo Rosa Chaves ${ }^{2}$ \\ Luíza Rosa Werneck ${ }^{3}$
}

Original

Paper

\begin{abstract}
Palavras-chaves: Resumo
Atendimento

Pré-hospitalar

Este artigo trata a participação da Enfermagem no Atendimento Pré-Hospitalar (APH), e têm como objeto de estudo os instrumentos normativos que orientam a prática de Enfermagem nesta área.
\end{abstract}

Enfermagem

Instrumentos

Normativos

\section{Abstract}

This article intents to show the participation of the Nursing in Pre-Hospitalar Attendance, and has as study object the normative instruments that guide the Nursing practice on this area.

Key words:

Pre-Hospitalar Attendance

Nursing

Normative

Instruments
Submetido

em: 19/12/07

Revisado

em: 17/03/08

Aceito para

Publicação em: 25/03/08

\section{Introdução}

De acordo com Santos (2003) a realidade social dos nossos dias mostra que o trauma assumiu proporções endêmicas e, em muitas faixas etárias, tornou-se a principal causa de mortalidade, retirando da força produtiva da população um numeroso contingente, com prejuízo incalculável para a sociedade.

É responsabilidade de todos, inclusive do enfermeiro, criar e desenvolver no atendimento ao traumatizado, sistemas efetivos e eficazes para corresponder à expectativa de oferecer cada vez mais, melhores cuidados ao traumatizado. Devido à necessidade de um atendimento inicial da vítima traumatizada, ainda na via pública, iniciou-se em 1981, no Rio de Janeiro, o APH (Atendimento PréHospitalar).

O APH é uma especialidade da Enfermagem, que surge junto com um crescente número de serviços de emergência pré-hospitalar móvel em todo o Brasil, através de empresas de concessões rodoviárias, prefeituras municipais e grupos privados. E nessa mesma direção de ofertas de serviços, cresce o número de atendimentos, uma demanda acompanhada pela modernidade do cotidiano,

\footnotetext{
${ }^{1}$ Coordenadora e professora dos cursos de Pós-graduação em Enfermagem em Cardiologia e Emergência Pré-hospitalar e Intra-hospitalar do UniFoa; Professora titular da graduação em enfermagem do UniFoa; Doutoranda da EEAN/UFRJ; Mestre em Enfermagem pela UNIRIO; Especialista em Enfermagem Intensivista pela UERJ.

${ }^{2}$ Professor dos cursos de Pós-graduação em Enfermagem em Cardiologia e Emergência Pré-hospitalar e Intra-hospitalar do UniFoa; Especialista em Enfermagem em Cardiologia pela Escola de Enfermagem Anna Nery/UFRJ; Enfermeiro da Nova Dutra.

${ }^{3}$ Enfermeira da Unidade de Emergência do Hospital São Camilo (VR); Especializanda do curso de pós-graduação em Enfermagem em Emergência Pré-hospitalar e Intra-hospitalar do UniFoa.
} 
que no retrocesso do desenvolvimento envolve vidas humanas em acidentes de trânsito, atropelamentos, violência (agressões, quedas etc.) e, emergências clínicas diversas.

Bitencourt e Figueiredo (2001) dizem ter:

“... neste final de século o cuidado está mais para ser executado fora dos como nível pré-hospitalar móvel na área de urgência, atendimento que procura chegar ambientes hospitalares (...) parece ser uma Enfermagem de e na rua, aquela enfermagem para um cliente especial - que está em risco de vida entre viver e morrer".

O APH tem como característica ser um serviço de utilidade pública, que está em ascensão em todo o Brasil, regulado pelo Ministério da Saúde (2001) que o define como $o$ atendimento que chega precocemente à vítima, após ter ocorrido um agravo à sua saúde (de natureza traumática ou não traumática ou ainda, psiquiátrica) que possa levar o sofrimento, seqüelas ou mesmo a morte, sendo necessário, portanto, prestar-lhe atendimento e/ou transporte adequado a um serviço devidamente hierarquizado e integrado ao Sistema Único de Saúde.

Nas faculdades, ainda não existem disciplinas que preparem os enfermeiros para este tipo de atendimento e conseqüentemente o aprendizado vai se fazendo, no dia-a-dia, sem orientação adequada. Estes profissionais contam principalmente com o seu esforço pessoal. Porém, devemos ter uma visão e ação desapaixonada em certos momentos, e tomarmosconsciência através do conhecimento sobre tudo o que nos envolve no APH.

Várias medidas foram apresentadas por organizações competentes e representantes da área de saúde, através de portarias de governo, diretrizes e resoluções de entidades de classes, com o objetivo de nortearem o APH. Os instrumentos normativos fornecem informações para a prática, os aspectos legais, ético e profissional que acrescentados à assistência torna a Enfermagem melhor capacitada para função.

Dentre as inúmeras possibilidades de destaque para o desenvolvimento de um estudo complexo e, que comporta muitas variáveis, como por exemplo razões da não inclusão desta temática na grade curricular da graduação em Enfermagem, capacitação da equipe de Enfermagem que atua com esta clientela, dentre outras. No entanto, optou-se por contemplar aspectos que dão ênfase ao conhecimento dos profissionais de Enfermagem que atuam no APH sobre instrumentos normativos.

O estudo justifica-se pela necessidade de divulgar para toda comunidade acadêmica, profissionais de saúde e setores de salvamento (socorristas, bombeiros e policiais), os instrumentos normativos à prática profissional no APH, e a atuação da Enfermagem neste tipo de atendimento.

Esta área especializada vem ofertando um mercado de trabalho mais abrangente para o profissional de Enfermagem, através de concursos públicos para as corporações de bombeiros, com a implementação do governo federal na atenção integral às urgências $\mathrm{e}$ emergências com envolvimento do APH, através do Serviço de Atendimento Médico de Urgência (SAMU), em parceria com os municípios. As empresas de concessões rodoviárias, também estão se enquadrando para as exigências legais, abarcando também um grande número de profissionais de Enfermagem.

Observando que o APH tornouse assunto de atenção integral no sistema de atendimento a saúde e, é um mercado emergente para os profissionais de saúde interessados na área de urgências, onde a oferta de trabalho por meio de concursos públicos cresce rapidamente. Portanto, um estudo que destaque a atuação da Enfermagem nesta área especializada, é relevante.

O estudo poderá contribuir para o melhor entendimento sobre APH, sua importância para o profissional em formação que busca essa disciplina nas escolas de enfermagem e não encontra, onde a prática de um cuidar, com visão assistencial e legal torna-se fundamental para a construção dos conhecimentos em APH. Para a produção científica esse estudo inicia, juntamente com os poucos descritos sobre o tema, a divulgação 


\section{Principais Instrumentos Normativos do} APH

Muitos caminhos e direções orientaram a prática do $\mathrm{APH}$, sobre tudo as atividades de socorro exercidas por corporações de bombeiros, levando em 1998 o Conselho Federal de Medicina (CFM) publicar parecer N. 1529/98 que trata sobre normas e procedimentos para o APH (CFM, 1998). Este instrumento define a regulação, os recursos humanos necessários (dentre eles a enfermagem) sua competência e os equipamentos. A elaboração deste documento ter sido constituída por um grupo de vinte (20) entidades envolvidas com o APH, com a observação de que nenhum era da enfermagem, apesar de tratarem sobre regulação médica por radiotelefonia e profissionais envolvidos, (competências e perfil profissional de auxiliar e técnico de Enfermagem e enfermeiros).

Em 1999, o Ministério da Saúde (MS) publicou portaria 824/GM em 24/06/99 que trata sobre normatização do APH a nível nacional sendo esta portaria baseada no parecer do CFM anteriormente descrito.

“... é fato que a portaria do MS tem origem na resolução do CFM, pois incorporou na íntegra o conteúdo da resolução do CFM".(MARTINS \& PRADO 2003)

Em 23 de fevereiro de 2000, o Conselho Federal de Enfermagem publica a resolução N. 225 que dispõe sobre o cumprimento de prescrição medicamentosa / terapêutica à distância.

Resolve autorizar a execução de prescrição médica através de rádio, telefonia e meios eletrônicos em situações de emergência, na qual, haja iminente e grave risco de vida do cliente. Sendo necessário que o profissional de Enfermagem elabore relatório descrevendo o acontecimento.

Em 22 de março de 2001, o Conselho Regional de Enfermagem de São Paulo - SP, publicou a DIR 001/2001 homologado pelo COFEN através de decisão 021/2001 em 3 de básico e suporte avançada de vida.

Decide que todo procedimento de APH de suporte básico ou avançado seja incondicionalmente prestado por enfermeiros, técnicos e auxiliares de enfermagem. Que o profissional envolvido no APH comunique ao conselho de acordo com o código de ética dos profissionais de Enfermagem, toda situação em que não haja a atuação do enfermeiro (COFEN, 2001).

Compete privativamente ao enfermeiro: dirigir, coordenar, planejar, prescrever, delegar, supervisionar e auxiliar nas ações de Enfermagem. Os procedimentos de alta complexidade deverão exclusivamente ser executado por enfermeiros. A assistência de Enfermagem em UTI móvel e suporte avançado de vida (terrestre, aérea ou aquática) deverá ser prestada pelo enfermeiro. Ao auxiliar de Enfermagem será permitida somente assistência básica, não completa. $\mathrm{O}$ enfermeiro deverá implementar e documentar a sistematização da assistência de Enfermagem. Admite-se aos militares das forças armadas, bombeiros e policia militar desde que treinados e sob ação da instituição a execução de suporte básico.

Em $1^{\circ}$ de junho de 2001, o Ministério da Saúde publicou a portaria N. 814/GM, revogando a portaria N.824/99. Neste documento é destaque os princípios do Sistema Único de Saúde (SUS) que são (universalidade de acesso, integralidade na atenção e eqüidade). Envolve a telemedicina, define equipe de Enfermagem e outros profissionais, veículos, equipamentos, medicamentos. Este instrumento considera a responsabilidade do SUS de instrumentalizar e estimular a implantação de Serviços de APH.

Em de 12 de julho de 2001, o Conselho Federal de Enfermagem publicou a Resolução N.260/2001 que descreve as especialidades deEnfermagem, decompetência do enfermeiro, total de trinta e sete (37) especialidades, entre elas o atendimento préhospitalar. Esta resolução foi revogada pela Resolução COFEN N.290/2004, publicada em 24 de março de 2004, já constituindo 42 especialidades. 
Em 5 de novembro de 2002, o Ministério da Saúde publicou a portaria 2048/GM que é o Regulamento técnico dos sistemas estaduais de urgência e emergência.

Este instrumento normativo descreve o APH em seu capitulo IV. Considera que as urgências não se constituem em especialidade médica ou de Enfermagem e que nos cursos de graduação a atenção dada à área ainda é bastante insuficiente. Este documento consolida todas as portarias anteriormente publicadas, mas trata o APH já devidamente integrado ao Sistema Estadual de Urgência e Emergência e não mais isoladamente, apresenta os principais pontos para a enfermagem: prazo de dois anos para adaptação à norma, que será em novembro de 2004. Ressaltando que a presença do Enfermeiro assumindo o que lhe compete legal e tecnicamente deve ser inserido desde a sua regulação em portarias anteriores.

O enfermeiro é responsável pelas atividades de Enfermagem. Enfermeiros assistenciais: enfermeiro responsável pelo atendimento de enfermagem necessário para a reanimação e estabilização do paciente, no local do evento e durante o transporte. Auxiliar e técnico de Enfermagem: atuação sob supervisão imediata do profissional enfermeiro. Ministrar medicamento por via oral e parenteral mediante prescrição do médico regulado por telemedicina.

Define tipos de veículos destinados ao APH-móvel, estabelecendo estruturas mínimas para cada tipo.

Esta portaria cria também os Núcleos de Educação em urgências:

Destinados à capacitação dos recursos humanos envolvidos na área, sendo certificador oficial, cria diretrizes curriculares mínimas aos cursos de capacitação de recursos humanos (RH) em todas as áreas profissionais de atuação no setor.

Técnicos e Auxiliares de Enfermagem: 180 horas, sendo 47 teóricas e 133 horas práticas. Enfermeiro: 160 horas, sendo 60 teóricas e 100 horas práticas.

Os Núcleos de Educação em Urgência devem se organizar como espaços de saber interinstitucional de formação, capacitação, habilitação e educação continuada.

O Conselho Regional de Enfermagem de São Paulo, no uso de suas atribuições legais e regimentais, tendo em vista a deliberação do Plenário em sua Reunião Ordinária nº 520a, realizada em 06 de março de 2.001 .

CONSIDERANDO, a Constituição da República Federativa do Brasil, art. 50 inciso II "ninguém será obrigado a fazer ou deixar de fazer alguma coisa senão em virtude de lei";

CONSIDERANDO, o Decreto-Lei 2.848, de 07 de dezembro de 1940, Código Penal, art. 135, que trata da " omissão de socorro";

CONSIDERANDO, os termos da Lei 5.905, de 12 de julho de 1973, que determina ao Conselho Federal de Enfermagem e aos Conselhos Regionais de Enfermagem a normatização do exercício das atividades de enfermagem;

CONSIDERANDO, os termos da Lei 7.498, de 25 de junho de 1986, que regulamenta o exercício profissional;

CONSIDERANDO, a necessidade e a obrigatoriedade legal e ética deste Conselho em assegurar uma assistência de Enfermagem isenta de riscos à integridade e à vida das vítimas/pacientes/clientes que são assistidos em situações de urgência/emergência relacionadas com o assunto em questão;

CONSIDERANDO, a existência de situações de extremo risco de vida e integridade à saúde que tem sido constatada, no Estado de São Paulo, por este Conselho, nas situações de urgência/emergência relacionadas com o APH e com o suporte básico e Avançado de Vida;

CONSIDERANDO, os termos da Decisão proferida pela 1a. Vara Federal do Distrito Federal, que vetou a criação e excluiu a figura do "SOCORRISTA";

\section{RESOLVE:}

Artigo $1^{\circ}$ - Que o Atendimento Pré- 
Hospitalar, de Suporte Básico e de Suporte Avançado de Vida, em termos de procedimentos de Enfermagem previstos em Lei sejam, incondicionalmente, prestados por Enfermeiros, Técnicos de Enfermagem ou Auxiliares de Enfermagem, observados os dispositivos constantes na Lei 7498/86 e decreto-lei 94.406/87;

Artigo $2^{\circ}$ - Que o profissional de Enfermagem que esteja envolvido com este tipo de assistência, comunique ao COREN-SP, de acordo com o determinado pelo Código de Ética dos Profissionais de Enfermagem, todas as situações em que não estiver sendo delegado, supervisionado e acompanhado pelo ENFERMEIRO na execução dos procedimentos de Enfermagem pertinentes à situação que se apresente;

Parágrafo primeiro - Compete privativamente ao Enfermeiro, Dirigir, Coordenar, Planejar, Prescrever, Delegar, Supervisionar e Avaliar as ações de Enfermagem, de acordo com o nível de dependência das vítimas/pacientes/clientes, quando o mesmo delegará, se a assistência deverá ser feita por Técnico e/ou Auxiliar de Enfermagem;

Parágrafo segundo - No que tange aos procedimentos de Enfermagem considerados de alta complexidade, estes deverão ser realizados exclusivamente pelos profissionais Enfermeiros.

Parágrafo terceiro - A Assistência de Enfermagem em unidades móveis de UTI e Suporte Avançado de Vida (terrestre, aéreo ou aquático), deverá ser prestada pelo ENFERMEIRO, de acordo com o determinado pela Lei 7.498/86 e pelo Decreto-Lei 94.406/87;

Parágrafo quarto - Ao Auxiliar de Enfermagem será permitida somente a assistência básica, não complexa, de acordo com os termos da lei vigente. Havendo risco iminente ou complexidade na assistência, esta deverá ser prestada somente pelo Enfermeiro;

Artigo $3^{\circ}$ - Todos os profissionais de enfermagem deverão ser cadastrados na empresa e a listagem atualizada deverá ser enviada ao COREN-SP para verificação da situação de Inscrição profissional junto ao Conselho;
Artigo $4^{\circ}$ - O ENFERMEIRO deverá implementar e documentar a Sistematização da Assistência de Enfermagem, através do registro das informações técnicas colhidas (protocolo de atendimento), contendo o Histórico de Enfermagem, Prescrição e Evolução da Assistência de Enfermagem determinada pelo mesmo

Parágrafo único - $\mathrm{O}$ registro contendo a Evolução da Assistência de Enfermagem deverá abranger todo o Atendimento prestado, até o momento em que o paciente/ vítima estiver sob responsabilidade do serviço ao qual foi transferido;

Artigo $5^{\circ}$-TodoprocedimentodeEnfermagem que estiver sendo executado por leigos ou por quem não seja profissional de Enfermagem, deverá ser comunicado imediatamente a este Conselho, para as necessárias medidas legais pertinentes, por Exercício Ilegal da Profissão, de acordo com o disposto no Art. 47 da Lei das Contravenções Penais;

Parágrafo único - Admitem-se aos militares das Forças Armadas, Bombeiros e Policiais Militares das Forças Auxiliares, enquanto investidos na função de militar junto à guarnição, e desde que treinados para atuar em situações de RESGATE, a execução de quaisquer procedimentos essenciais ao Suporte Básico de Vida, à preservação da vida e integridade das vítimas/pacientes/ clientes, em situações de urgência/ emergência, até que seja possível o acesso pelo profissional de Saúde;

Artigo $6^{\circ}$ - O profissional Enfermeiro é responsável pelas vítimas/pacientes/clientes, em termos de Assistência de Enfermagem, durante todo o período em que estiver sob seu atendimento de urgência/emergência;

Parágrafo único - No caso de REMOÇÃO, por ser procedimento possível de planejamento e programação, deverá o ENFERMEIRO avaliar se deverá ser feita pelo Técnico ou Auxiliar de Enfermagem, considerando-se as respectivas competências legais;

Artigo $7^{\circ}$ - O ENFERMEIRO que responder pela Instituição/unidade/posto de Atendimento Pré-Hospitalar, Suporte Básico e Suporte Avançado de Vida, deverá providenciar seu registro como Responsável 
Técnico de Enfermagem neste COREN-SP, obtendo com isso, o respectivo Certificado de Responsável Técnico de Enfermagem;

Artigo $8^{\circ}$ - Todas as situações não previstas nesta DECISÃO serão apreciadas à luz da Lei por este Conselho, com a participação dos profissionais de Enfermagem envolvidos na mesma;

Artigo $9^{\circ}$ - Esta decisão entrará em vigor na data de sua publicação, revogando-se todas as disposições em contrário.

(Homologado pelo Conselho Federal de Enfermagem através da Decisão COFEN $N^{\circ}$ 021/2001 de 03/04/2001).

\section{Considerações Finais}

A participação da Enfermagem no APH vem se constituindo ao passo do surgimento desta "especialidade", que caminha numa vertente de crescimento e importância desde o final do século XX (década de 80) e seus instrumentos normativos começam a surgir em 1998. A Enfermagem está envolvida nesse processo, e sua instrumentalização se transforma em ferramenta importante nas atividades profissionais.

Deve-se resaltar a necessidade da participação das escolas de Enfermagem nos níveis de formação técnica, universitário, e pós-graduação em elaborar proposta curricular, incluindo o $\mathrm{APH}$, porque entendo que a sala de aula é a base fundamental para o entendimento de toda especialidade.

Como o tema tornou-se notório nos diversos seguimentos da área da saúde, e mediante a queixa da ausência da disciplina nas grades curriculares, fica a proposta para os enfermeiros atuantes e incluídos no APH, que realizem planejamento de educação continuada, inserindo os instrumentos normativos à prática de Enfermagem no $\mathrm{APH}$ na abrangência educacional a Técnicos e auxiliar de Enfermagem envolvida nesse tipo de atendimento especial.

\section{Bibliografia}

BITENCOURT, A. \& FIGUEIREDO, N. Enfermeiras (os) na rua fazendo cuidados críticos: o saber fazer na fronteira entre a vida e a morte. Revista de pesquisa: cuidado é fundamental. Uni-Rio. Rio de Janeiro 2001 Jan/jun V.N.1: 80-82.

\section{CONSELHOFEDERALDEENFERMAGEM}

- Resolução COFEN n²25/2000: Dispõe sobre o cumprimento de prescrição medicamentosa/ terapêutica a distancia. Rio de Janeiro, 2000. Disponível em: <http://www.corensp.org.Br/ resoluções $>$ acessado em 15 mar.2004.

\section{CONSELHOFEDERALDEENFERMAGEM}

- Resolução COFEN $n^{\circ}$ 260/2001: Especialidades de enfermagem. Rio de Janeiro, RJ. 2001. Disponível em: <http:// www.corensp.org.br/resoluções $>$ acessado em 15 mar2004.

\section{CONSELHOFEDERALDEENFERMAGEM}

- Resolução COFEN no. 290/2004: Especialidades de Enfermagem. Rio de Janeiro, RJ. 2004. Disponível em: <http://www. corensp.org.com.br/resoluções $>$ acessado em 30 set2004.

CONSELHO FEDERAL DE MEDICINA. Resolução $n^{\circ}$ 1529/98: Normatização do Atendimento pré-hospitalar. Brasília, DF. 1998. Disponível em: <http://www.aph.com. br/legislação>. acessado em 11 fev.2004.

CONSELHO REGIONAL DE ENFERMAGEM. Decisão COREN-SP DIR 01/2001: Regulamenta as Atividades de Enfermagem no Atendimento pré-hospitalar. São Paulo. 2001. (Homologado COFEN através da decisão COFEN n ${ }^{\circ}$ 021/2001 em 03/04/2001).Disponível em: <http://www. corensp.org.br/decisões $>$ acessado em 15 mar2004.

FONTENELE, K. J. \& SARQUIS, S. I. J. S. Urgência e Emergência em Enfermagem. Goiânia - GO: Cultura e Qualidade 2004.

KURCGANT, P. \& Cols. Administração em Enfermagem. São Paulo, EPU; 1991.

MARTINS, P. P. S \& PRADO, M. L. ENFERMAGEM E SERVIÇO DE ATENDIMENTO PRÉ-HOSPITALAR - dos caminhos e perspectivas. Revista Brasileira de Enfermagem, Brasília, DF. 2003; jan/fev 56(1): 71.75 . 
MINISTÉRIO DA SAÚDE (BR) - Portaria $n^{\circ}$

824/GM de 24 de julho de 1999: Normatização do Atendimento Pré-hospitalar e Transporte Hospitalar no Brasil. Brasília, DF. 1999. Disponível em: <http://www.aph.com.br/ portaria $>$. Acessado em 11 fev.2004.

MINISTÉRIO DA SAUDE (BR) - Portaria $n^{\circ} 737 / G M$ de 16 de maio de 2001: Política Nacional de Redução de Morbimortalidade por Acidentes e Violência. Brasília, DF. 2001. Disponível em: <http://www.aph.com.br/ portaria $>$ acessado em 20 fev.2004.

MINISTÉRIO DA SAUDE (BR) - portaria n'814/GM de 01 de junho de 2001: Normatização do Atendimento Pré-hospitalar Nível de Urgência no Brasil. Brasília, DF. 2001. Disponível em: <http://www.aph.com. $\mathrm{Br} /$ portaria $>$ acessado em 20 fev.2004.

MINISTÉRIO DA SAUDE (BR) - Portaria $n^{\circ}$ 1864/GM de 29 de setembro de 2003: Implantação de Serviços de Atendimento Móvel de Urgência - Municípios e regiões de todo território brasileiro: SAMU -192 . Brasília. DF. 2003.Disponível em <http:// dtr2001.saude.gov.br/samu $>$ acessado em 10 fev.2004.

MORGAN, G. - Imagens da Organização. 2a ed. São Paulo, Atlas; 2002.

RODRIGUES, M. S. P. \& LEOPARDI, M. T. - O Método de Análise de Conteúdo: Uma versão para Enfermeiros . Fortaleza, Graficeler;1999.

SANTOS, R. R. et al - Manual de Socorro de

Emergência. São Paulo, Atheneu; 2003.

Informações bibliográficas:

Conforme a NBR 6023:2002 da Associação Brasileira de Normas Técnicas (ABNT), este texto científico publicado em periódico eletrônico deve ser citado da seguinte forma:

BRAZ, M. R.; CHAVES, R. R.; WERNEK, L. R.. Instrumentos Normativos no Atendimento PréHospitalar: enfermagem e legislação. Cadernos UniFOA, Volta Redonda, ano 3, Edição Especial, maio. 2008. Disponível em: <http://www.unifoa.edu.br/pesquisa/caderno/especiais/pos-graduacao/01/59.pdf> 